\title{
Identifying the Most Appropriate Classifier for Underpinning Assistive Technology Adoption for People with Dementia: An Integration of Fuzzy AHP and VIKOR Methods
}

Miguel Ortíz-Barrios, Chris Nugent, Matias García-Constantino, and Genett Jimenez-Delgado

\begin{abstract}
Recently, the number of People with Dementia (PwD) has been rising exponentially across the world. The main symptoms that PwD experience include AQ1 impairments of reasoning, memory, and thought. Owing to the burden faced by this chronic condition, Assistive Technology-based solutions (ATS) have been prescribed as a form of treatment. Nevertheless, it is widely acknowledged that low adoption rates of ATS have hampered their benefits within a health and social care context. It is then necessary to effectively discriminate between adopters and nonadopters of such solutions to avoid cost implications, improve the life quality of adopters, and find intervention alternatives for non-adopters. Several classifiers have been proposed as advancement towards the personalisation of selfmanagement interventions for dementia in a scalable way. As multiple algorithms have been developed, an important step in technology adoption is to select the most appropriate classification alternative based on different criteria. This paper presents the integration of Fuzzy AHP (FAHP) and VIKOR to address this challenge. First, FAHP was used to calculate the criteria and sub-criteria weights under uncertainty and then VIKOR was implemented to rank the classifiers. A case study considering a mobile-based self-management and reminding solution for $\mathrm{PwD}$ is described to validate the proposed approach. The results revealed that Easiness of interpretation (GW $=0.192)$ and Handling of missing data $(\mathrm{GW}=$ o.145) were the two most important criteria. Furthermore, SVM $(Q j=1.0)$ and AB $(\mathrm{Qj}=0.891)$ were concluded to be the most suitable classifiers for supporting ATS adoption in PwD.
\end{abstract}

Keywords: Technology adoption · Dementia · Fuzzy Analytic Hierarchy

Process (FAHP) · VIKOR · Healthcare 